\title{
Poly(ADP-ribose) engages the TDP-43 nuclear-localization sequence to regulate granulo- filamentous aggregation
}

Leeanne McGurk $^{1 \#}$, Edward Gomes ${ }^{2 \#}$, Lin Guo ${ }^{2 \#}$, James Shorter $^{2 *}$, and Nancy M. Bonini ${ }^{1 *}$.

\author{
Affiliations \\ ${ }^{1}$ Department of Biology, University of Pennsylvania, Philadelphia, PA 19104, USA \\ ${ }^{2}$ Department of Biochemistry and Biophysics, Perelman School of Medicine, University of \\ Pennsylvania, Philadelphia, PA 19104, USA \\ \# co-first authors \\ *co-corresponding authors.
}




\section{A}

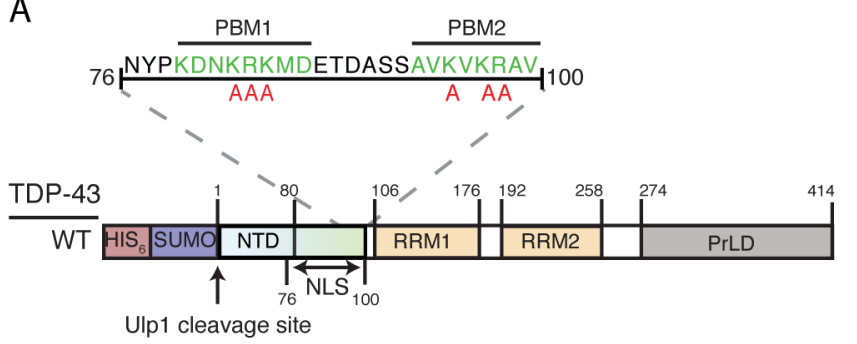

PBM mutation
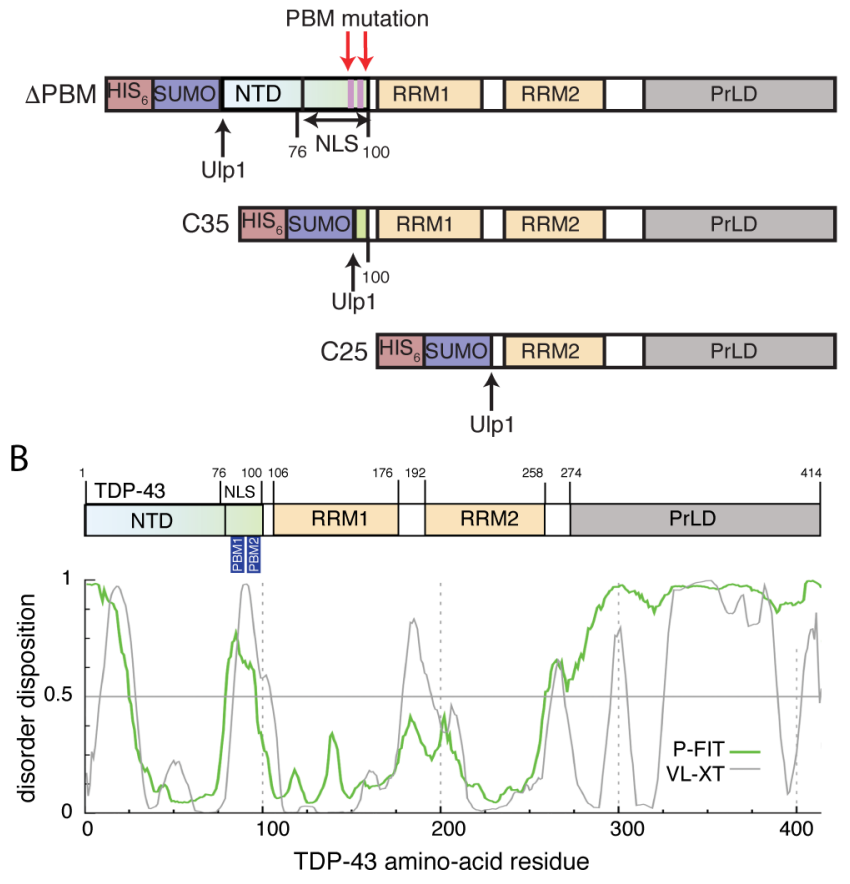

Figure S1. TDP-43 domain architecture.

A. Schematic of TDP-43 constructs used in this study. The Ulp1 cleavage site is downstream of the $\mathrm{HIS}_{6}$-SUMO-tag and upstream of the TDP-43 ATG-start codon in all constructs. The amino acids in green are the PAR-binding motifs (PBM1 and PBM2), and the red amino acids represent the mutations made to generate TDP-43- $\triangle$ PBM. NTD: N-terminal domain. NLS: Nuclear localization sequence. PBM: PAR-binding motif. RRM: RNA recognition motif. PrLD: prion-like domain.

B. Schematic of the domain architecture of TDP-43 and predicted disorder. Disorder was predicted using an online database with the VL-XT and P-Fit (PONDR-Fit) algorithms. ${ }^{1-3}$ 
A

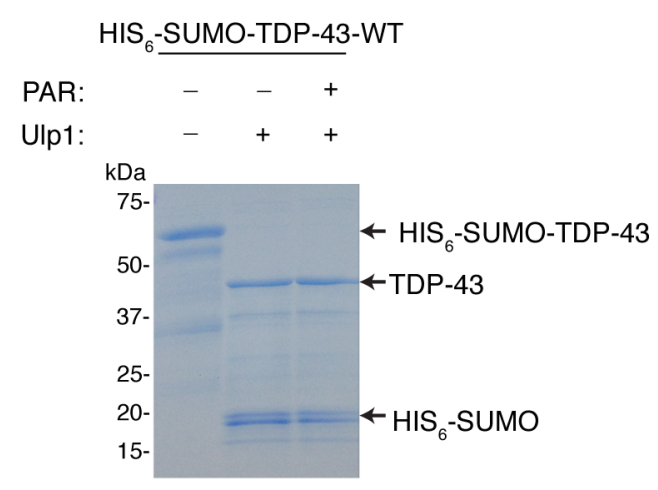

B

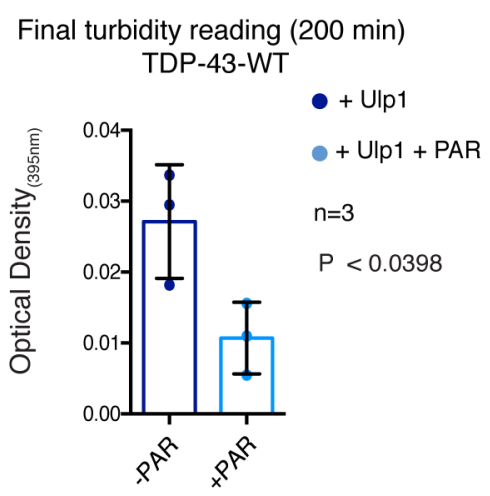

$\mathrm{C}$

Turbidity of TDP-43-WT

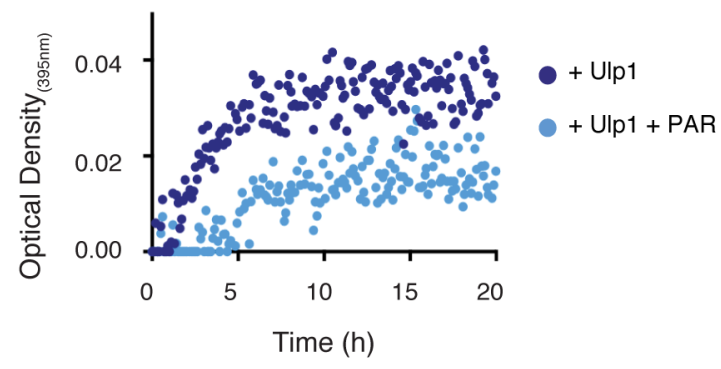

Figure S2: PAR reduces TDP-43 aggregation in vitro.

A. Incubation of Ulp1 with purified $10 \mu \mathrm{M}$ SUMO-TDP-43-WT results in cleavage of the Nterminal $\mathrm{HIS}_{6}-\mathrm{SUMO}$-tag to produce TDP-43-WT and $\mathrm{HIS}_{6}-\mathrm{SUMO}$ at the expected molecular weights. PAR at substoichiometric amounts $(6 \mu \mathrm{M}$ equivalents to mono(ADP-ribose) $)$ had no effect on Ulp1-cleavage of $10 \mu \mathrm{M} \mathrm{HIS}_{6}$-SUMO-TDP-43-WT. The final turbidity samples (200 min) were processed for SDS-PAGE and visualized by Coomassie blue staining. 
B. PAR, at a concentration of $6 \mu \mathrm{M}$ equivalents to mono(ADP-ribose), reduces the final optical density measurement at $395 \mathrm{~nm}$ (200 min) of $10 \mu \mathrm{M}$ TDP-43-WT compared to PAR buffer control. The mean of the final $\mathrm{OD}_{395}( \pm \mathrm{SD})$ is presented. The $\mathrm{n}$ value is 3 independent experiments performed on two separate preparations of protein and demonstrates the reproducibility of the data. A two-tailed and unpaired T-test was performed.

C. Ulp1-cleavage of $10 \mu \mathrm{M}$ His $_{6}-\mathrm{SUMO}-\mathrm{TDP}-43-\mathrm{WT}$ at $30^{\circ} \mathrm{C}$ leads to an increase in optical density at $395 \mathrm{~nm}$ that plateaus after 10-20 h. Co-incubation with PAR at $6 \mu \mathrm{M}$ equivalents to mono(ADP-ribose) reduces the amplitude of the plateau phase, indicating that PAR reduces TDP43 aggregation. Graph is a representative dataset. 


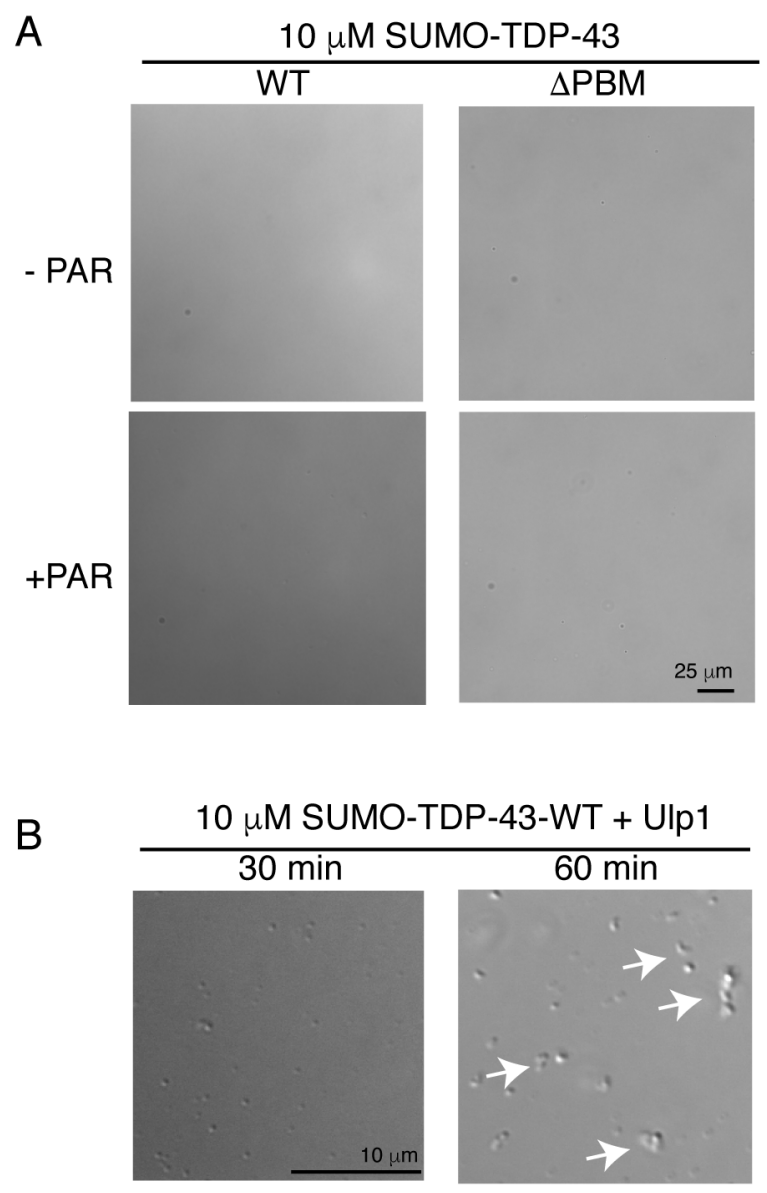

Figure S3. Examination of cleaved TDP-43 by optical microscopy.

A. SUMO-TDP-43-WT and SUMO-TDP-43- $\triangle \mathrm{PBM}$ in $70 \mathrm{mM} \mathrm{NaCl}$ remained diffuse as examined by differential interference contrast (DIC) microscopy.

B. Ulp1-cleaved SUMO-TDP-43-WT in 70mM NaCl forms spherical structures that after 60 min coalesce into irregular solid structures (arrows). 


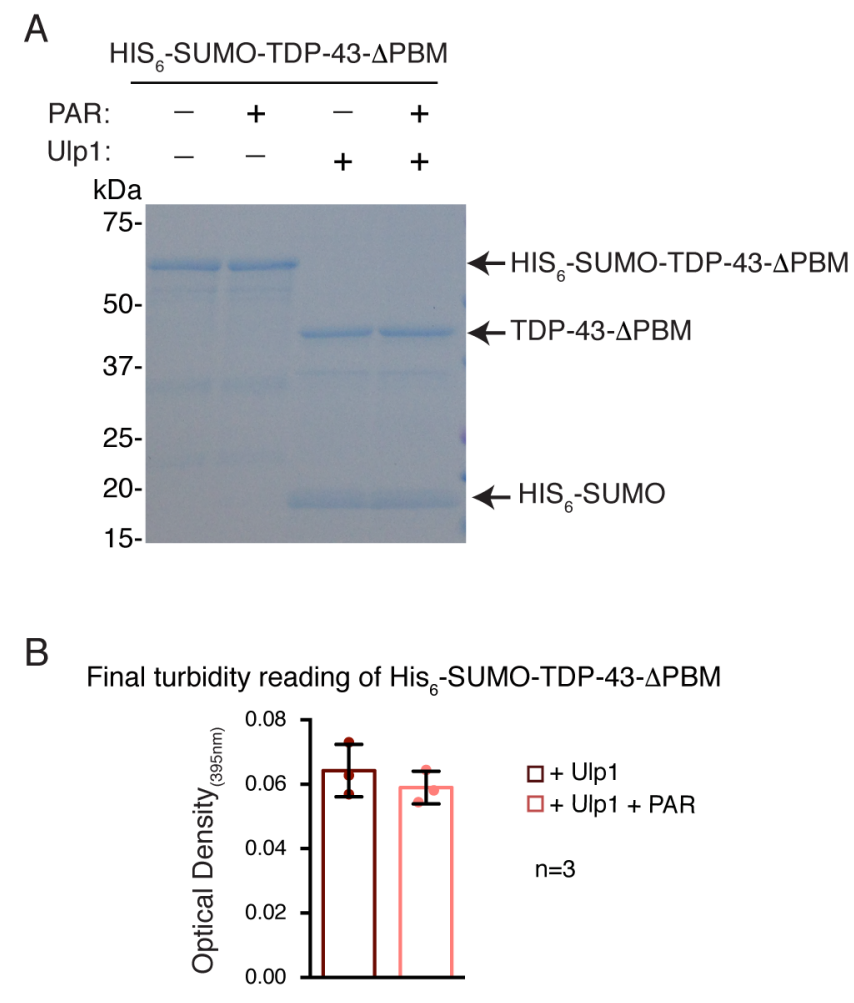

Figure S4. The PAR-binding motifs are required for PAR-mediated inhibition of TDP-43 aggregation.

A. Incubation of Ulp1 with purified $\mathrm{HIS}_{6}-\mathrm{SUMO}_{\mathrm{TDP}}-43-\triangle \mathrm{PBM}(10 \mu \mathrm{M})$ results in cleavage of the N-terminal $\mathrm{HIS}_{6}$-SUMO tag to produce TDP-43- $\triangle \mathrm{PBM}$ and $\mathrm{HIS}_{6}-\mathrm{SUMO}$ at the expected molecular weights. PAR at $6 \mu \mathrm{M}$ equivalents to mono(ADP-ribose) has no effect on Ulp1-cleavage of HIS $_{6}$-SUMO-TDP-43- $\triangle$ PBM $(10 \mu \mathrm{M})$. The final turbidity protein samples were processed for SDS-PAGE and visualized by Coomassie blue staining.

B. The final optical density measurement at $395 \mathrm{~nm}(54 \mathrm{~h})$ of $10 \mu \mathrm{M}$ SUMO-TDP-43- $\triangle \mathrm{PBM}$ is unaltered by the addition of PAR at a concentration of $6 \mu \mathrm{M}$ equivalents to mono(ADP-ribose). The mean of the final optical density reading at $395 \mathrm{~nm}( \pm$ SD.) from 3 independent experiments from 2 independent protein preparations is presented and demonstrates the reproducibility of the data. A two-tailed unpaired T-test was performed. The final turbidity readings were not significantly different $(P=0.3952)$. 
$10 \mu \mathrm{M} \mathrm{His}{ }_{6}$-SUMO-TDP-43-WT (endpoint of turbidity assay)

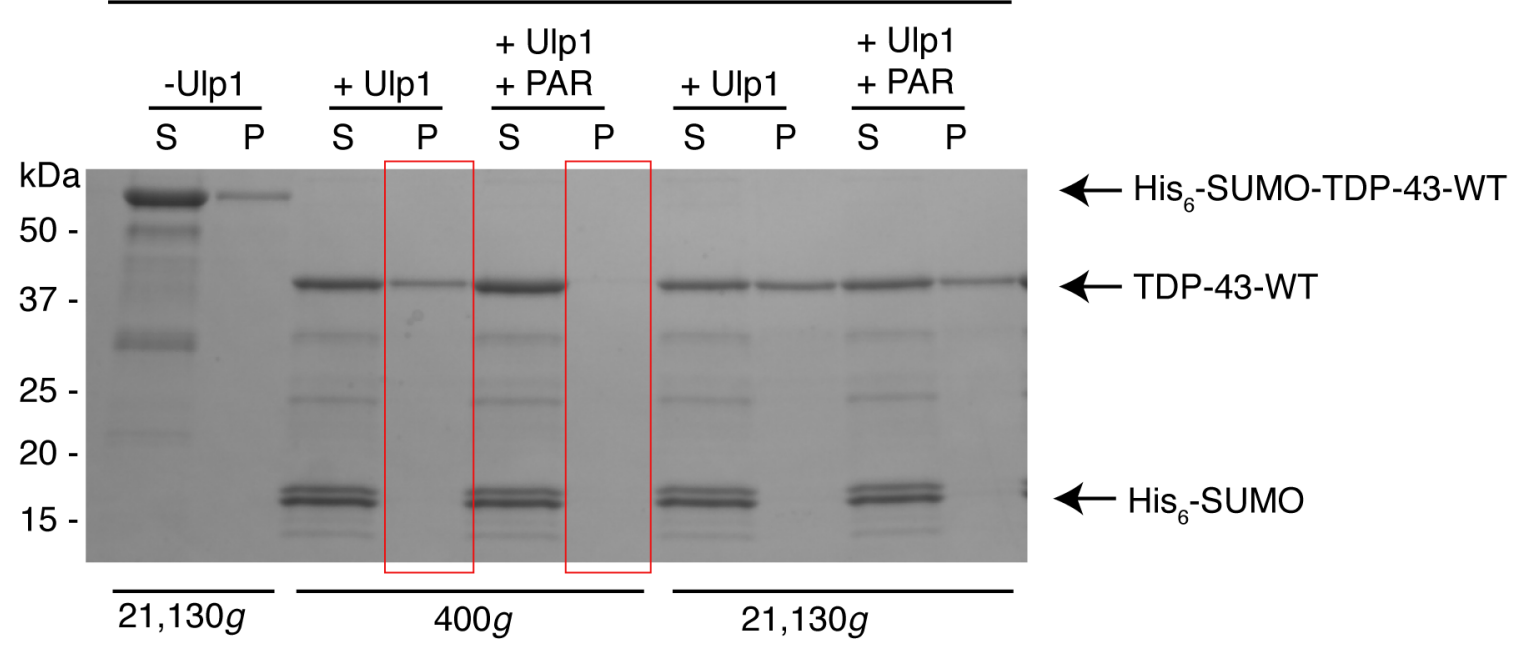

Figure S5. PAR inhibits formation of large TDP-43 aggregates.

HIS $_{6}$-SUMO-TDP-43-WT was subjected to a turbidity assay and the resulting protein was separated into the supernatant $(\mathrm{S})$ and the pellet fraction $(\mathrm{P})$, the protein samples were denatured and electrophoresed on an SDS-polyacrylamide gel. At $400 g$, only the large aggregates are pelleted, and in the presence of $6 \mu \mathrm{M}$ PAR the amount the TDP-43-WT in the pellet fraction (P) was reduced (compare red boxes), see Figure 3B for quantification. At 21,130g, large and small TDP-43 aggregates are pelleted and the presence of PAR had no effect on the amount of TDP-43WT in the insoluble pellet. For quantification see Figure 3C. Consistent with the TEM analysis (see Figure 3A-B), these data indicate that PAR inhibits the formation of large TDP-43 aggregates. 
A

\begin{tabular}{ccccccc} 
& \multicolumn{4}{c}{$1 \mu \mathrm{M} \mathrm{HIS}_{6}$-SUMO-TDP-43 } & & $\alpha$-Syn \\
\cline { 2 - 5 } & WT+Ulp1 & C25 -Ulp1 & C25 +Ulp1 & $(1 \mu \mathrm{M})$ & Buffer \\
\hline ThT fluorescence $(\mathrm{AU})$ & 297 & 296 & 204 & 2479 & 261 \\
\hline
\end{tabular}

B

\begin{tabular}{ccccccc} 
& \multicolumn{3}{c}{$10 \mu \mathrm{M}_{\mathrm{HIS}}$-SUMO-TDP-43 } & & \\
\cline { 2 - 5 } & WT+Ulp1 & C25 -Ulp1 & C25 +Ulp1 & $(1.8 \mu \mathrm{M})$ & Buffer \\
\hline ThT fluorescence $(\mathrm{AU})$ & 676 & 277 & 683 & 3640 & 207 \\
\hline
\end{tabular}

Figure S6. TDP-43-C25 aggregates were not reactive to Thioflavin T.

A. TDP-43-WT and TDP-43-C25 (both $1 \mu \mathrm{M}$ ) at the endpoint of a turbidity assay were tested for reactivity to $10 \mu \mathrm{M}$ Thioflavin $\mathrm{T}$ (ThT) excited at $440 \mathrm{~nm}$. Emission was collected at $482 \mathrm{~nm}$. While the positive control, $\alpha$-Synuclein ( $\alpha$-Syn) fibrils, was reactive to ThT, TDP-43-WT, HIS $6^{-}$ SUMO-TDP-43-C25 and TDP-43-C25 were no different to the buffer alone negative control. These data indicate that under these conditions these protein variants do not have amyloid-like properties.

B. At higher protein concentrations, TDP-43-WT, HIS 6 -SUMO-TDP-43-C25 and TDP-43-C25 remained non-reactive to $\mathrm{ThT}$. 


\section{METHODS}

\section{Plasmids}

Human TDP-43 (Uniprot IDL: Q13148), TDP-43-APBM, TDP-43-C35 and TDP-43-C25 subcloned into pE-SUMO (LifeSensors, Malvern, PA) are as described. ${ }^{4}$ Ulp1 was cloned into pFGET19 (Addgene \#64697). All plasmid inserts were fully sequenced and confirmed to be correct.

\section{PAR polymer}

Free PAR (commercially obtained from TREVIGEN (Gaithersburg, MD)) was synthesized from PARP-1 and ranged in size from 2-300 ADP-ribose subunits. Molar equivalencies were calculated by TREVIGEN. Briefly, the Absorbance of the PAR at $258 \mathrm{~nm}$ was divided by the extinction coefficient of ADP-ribose $\left(13,500 \mathrm{~cm}^{-1} \mathrm{M}^{-1}\right){ }^{5,6}$

\section{Purification of Recombinant His $_{6}$-SUMO-TDP-43 and aggregation assays}

HIS $_{6}$-SUMO N-terminally tagged TDP-43-WT, TDP-43- - PBM, TDP-43-C35 and TDP-43-C25 were purified as described. ${ }^{4}$ Yeast $\mathrm{HIS}_{6}$-Ulp1 protease (Ulp1, Uniprot ID: Q02724) was purified as described for His6-SUMO N-terminally tagged TDP-43-WT. ${ }^{4}$ Prior to the turbidity assay, protein was thawed and centrifuged at $16,100 \mathrm{~g}$ for $10 \mathrm{~min}$ to remove any preformed aggregates. Protein concentration was determined by Bradford assay (Bio-Rad, Hercules, CA). The kinetics of TDP-43 aggregation were assessed via turbidity assay by measuring the optical density at 395 $\mathrm{nm}$ at regular time intervals. Briefly, $10 \mu \mathrm{M}$ of the indicated protein was incubated in $50 \mathrm{mM}$ HEPES-NaOH (pH 7.5), 5\% glycerol, $70 \mathrm{mM} \mathrm{NaCl}, 5$ mM DTT, 6 mM TrisHCl (pH 8), $0.6 \mathrm{mM}$ EDTA with shaking at $30^{\circ} \mathrm{C}$ in Nunc 96-well optical bottom plates (Thermo Fisher Scientific, Waltham, MA) with PAR (Trevigen, Gaithersburg, MD) at a concentration of $6 \mu \mathrm{M}$ mono(ADPribose) equivalents in $10 \mathrm{mM}$ Tris- $\mathrm{HCl}(\mathrm{pH} 8)$ and $1 \mathrm{mM}$ EDTA, or equivalent volume of buffer (10 mM Tris-HCl [pH 8] and $1 \mathrm{mM}$ EDTA). At time 0, cleavage of the $\mathrm{HIS}_{6}$-SUMO tag was initiated with $1.8 \mu \mathrm{g}$ of Ulp1. Turbidity data are represented as the relative change, which was calculated by subtracting the initial $\mathrm{OD}_{395}$ value from the final $\mathrm{OD}_{395}$ value. 


\section{Transmission electron microscopy}

Transmission electron microscopy (TEM) of the aggregates was performed as described. ${ }^{7}$ Briefly, at the end point of the turbidity assay (200 min for TDP-43-WT and $54 \mathrm{~h}$ for TDP-43- $\Delta$ PBM) samples were diluted two-fold, $10 \mu \mathrm{L}$ of the diluted sample was adsorbed onto 300-mesh Formvar/carbon-coated copper grids (Electron Microscopy Sciences, Hatfield, PA) and stained with $2 \%(\mathrm{w} / \mathrm{v})$ uranyl acetate. Excess uranyl acetate solution was removed and the grids were dried. Samples were imaged using a FEI-Tecnai and a JEOL-1010T12 transmission electron microscope. For quantification, 2-3 micrographs representing each protein were analyzed by ImageJ. ${ }^{8} 1534-5829$ aggregates were quantified for each protein condition.

\section{Sedimentation assay}

HIS $_{6}$-SUMO-TDP-43-WT $(10 \mu \mathrm{M})$ was subjected to a turbidity assay and at the end of the 200min reaction, the resulting protein was separated into the supernatant fraction $(\mathrm{S})$ and the pellet fraction (P) by centrifugation for $10 \mathrm{~min}$ at $4^{\circ} \mathrm{C}$ with speed of $400 \mathrm{~g}$ or $21,130 \mathrm{~g}$. Supernatant and pellet fractions were then denatured in Laemmli buffer and $\beta$-mercaptoethanol, heat denatured at $99^{\circ} \mathrm{C}$. $11.4 \%$ of supernatant or pellet fraction was loaded and protein was resolved by sodium dodecyl sulfate polyacrylamide gel electrophoresis (SDS-PAGE) and stained with Coomassie Brilliant Blue, and the amount in either fraction (\% total) was determined by densitometry in comparison to known quantities of the SUMO-TDP-43-WT. Under these conditions, in the presence of Ulp1 and the absence of PAR, $\sim 50 \%$ of the TDP-43 remained in the supernatant fraction at both centrifugal speeds (Figure 3C).

\section{Thioflavin T assay}

HIS $_{6}$-SUMO-TDP-43-WT and HIS $_{6}$-SUMO-TDP-43-C25 $(10 \mu \mathrm{M})$ was aggregated in vitro as above. At the end of the 200-min reaction, protein was assessed without dilution or with dilution to $1 \mu \mathrm{M}$ using reaction buffer (50mM HEPES-NaOH (pH 7.5), $\%$ glycerol, $70 \mathrm{mM} \mathrm{NaCl}, 5 \mathrm{mM}$ DTT, $6 \mathrm{mM}$ TrisHCl (pH 8), $0.6 \mathrm{mM}$ EDTA). Thioflavin-T (ThT) was added into the reaction at $10 \mu \mathrm{M}$ and sample was excited at $440 \mathrm{~nm}$ with a $5 \mathrm{~nm}$ bandwidth. Fluorescence signal was collected at $482 \mathrm{~nm}$ with a $10 \mathrm{~nm}$ bandwidth. $\alpha$-Synuclein fibrils assembled as described ${ }^{9}$ in reaction buffer were used as a positive control. 


\section{Statistics}

All statistics were carried out using Graphpad prism 6 software.

\section{REFERENCES}

[1] Li, X., Romero, P., Rani, M., Dunker, A. K., and Obradovic, Z. (1999) Predicting Protein Disorder for N-, C-, and Internal Regions, Genome Inform Ser Workshop Genome Inform $10,30-40$.

[2] Romero, P., Obradovic, Z., Li, X., Garner, E. C., Brown, C. J., and Dunker, A. K. (2001) Sequence complexity of disordered protein, Proteins 42, 38-48.

[3] Xue, B., Dunbrack, R. L., Williams, R. W., Dunker, A. K., and Uversky, V. N. (2010) PONDR-FIT: a meta-predictor of intrinsically disordered amino acids, Biochim Biophys Acta 1804, 996-1010.

[4] McGurk, L., Gomes, E., Guo, L., Mojsilovic-Petrovic, J., Tran, V., Kalb, R. G., Shorter, J., and Bonini, N. M. (2018) Poly(ADP-Ribose) Prevents Pathological Phase Separation of TDP-43 by Promoting Liquid Demixing and Stress Granule Localization, Molecular cell 71, 703-717 e 709.

[5] Schultheisz, H. L., Szymczyna, B. R., and Williamson, J. R. (2009) Enzymatic synthesis and structural characterization of 13C, 15N-poly(ADP-ribose), J Am Chem Soc 131, 1457114578.

[6] Shah, G. M., Poirier, D., Duchaine, C., Brochu, G., Desnoyers, S., Lagueux, J., Verreault, A., Hoflack, J. C., Kirkland, J. B., and Poirier, G. G. (1995) Methods for biochemical study of poly(ADP-ribose) metabolism in vitro and in vivo, Anal Biochem 227, 1-13.

[7] Johnson, B. S., Snead, D., Lee, J. J., McCaffery, J. M., Shorter, J., and Gitler, A. D. (2009) TDP-43 is intrinsically aggregation-prone, and amyotrophic lateral sclerosis-linked mutations accelerate aggregation and increase toxicity, The Journal of biological chemistry 284, 20329-20339.

[8] Rueden, C. T., Schindelin, J., Hiner, M. C., DeZonia, B. E., Walter, A. E., Arena, E. T., and Eliceiri, K. W. (2017) ImageJ2: ImageJ for the next generation of scientific image data, BMC Bioinformatics 18, 529.

[9] Luk, K. C., Covell, D. J., Kehm, V. M., Zhang, B., Song, I. Y., Byrne, M. D., Pitkin, R. M., Decker, S. C., Trojanowski, J. Q., and Lee, V. M. (2016) Molecular and Biological Compatibility with Host Alpha-Synuclein Influences Fibril Pathogenicity, Cell Rep 16, 3373-3387. 